\title{
Clinical Features and Outcomes of 35 Disseminated Intravascular Coagulation Cases Treated with Recombinant Human Soluble Thrombomodulin at a Single Institution
}

\author{
Noriaki Kawano, ${ }^{1{ }^{*}}$ Shuro Yoshida, ${ }^{1)^{*}}$ Nobuyuki Ono, ${ }^{1)}$ Daisuke Himeji, ${ }^{1)}$ Yuri Nagahiro, \\ Sayaka Kawano, ${ }^{1)}$ Kiyoshi Yamashita, ${ }^{1)}$ Naoko Ikeda, ${ }^{1)}$ Shigehiro Uezono, ${ }^{1)}$ \\ Hidenobu Ochiai, ${ }^{3)}$ Fumiko Kawano, ${ }^{4)}$ Ikuo Kikuchi, ${ }^{1)}$ Fumihiko Ishikawa, ${ }^{5}$ \\ Kazuya Shimoda, ${ }^{6}$ Akira Ueda, ${ }^{1)}$ and Koichi Akashi ${ }^{7)}$
}

Disseminated intravascular coagulation (DIC) is a clinical entity with high mortality and is characterized by multiple organ failure caused by activation of systemic intravascular coagulation. Although a standard treatment for DIC has not been established owing to the absence of randomized controlled trials, recent reports have indicated that recombinant human soluble thrombomodulin (rTM) is effective against DIC. To elucidate the clinical characteristics and outcomes of DIC, we retrospectively analyzed 35 DIC patients treated with rTM at our institution over a 2-year period (infectious disease: 21 cases; hematological disease: 14 cases). Diagnosis of DIC was based on the diagnostic criteria for DIC of the Japanese Ministry of Health and Welfare. In addition to the treatment of underlying diseases, we administered rTM for 6 consecutive days. Twenty-one (60.0\%) of the DIC patients attained resolution of DIC at 7 days after administration (infectious disease: 61.9\%; hematological disease: $57.1 \%$ ). Furthermore, 7 of the remaining 14 DIC patients (who did not attain resolution at 7 days) attained resolution at an average of 12.1 days. Consequently, $28(80.0 \%)$ of the 35 patients were alive with resolution of DIC after a 28-day observation period (infectious disease: 76.2\%; hematological disease: 85.7\%). Among them, for 7 (70\%) of the 10 DIC patients with severe life-threatening bleeding symptoms without hemorrhagic shock, treatment with heparin was contraindicated; these patients were successfully treated with rTM without the progression of hemorrhage. In the majority of DIC patients, rTM administration may be an effective, safe, and feasible therapeutic modality producing a good outcome. $\lceil J$ Clin Exp Hematopathol 51(2) : 101-107, 2011]

Keywords: disseminated intravascular coagulation, recombinant human soluble thrombomodulin, infectious diseases, hematological diseases, DIC score (Japanese Ministry of Health and Welfare)

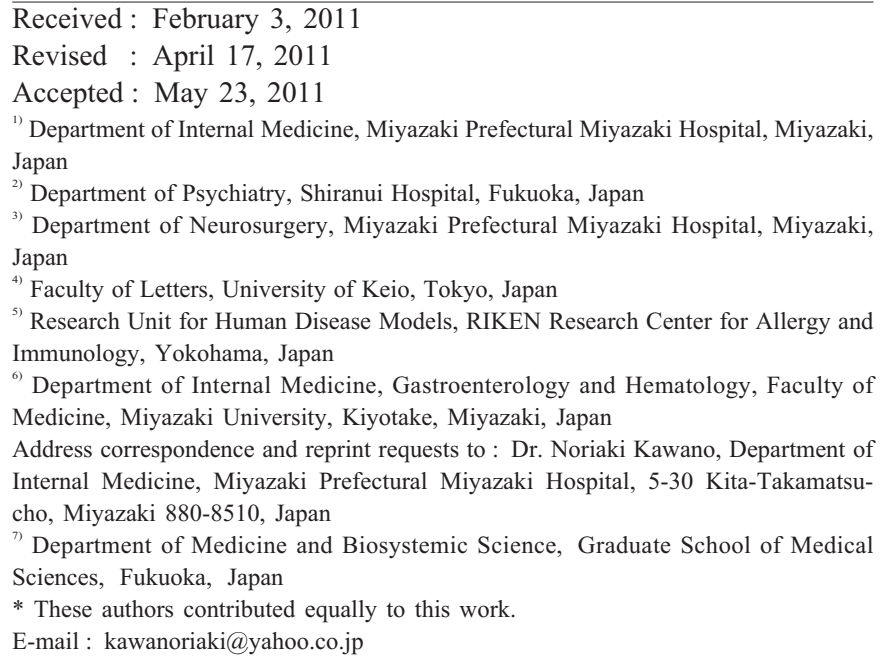

\section{INTRODUCTION}

Disseminated intravascular coagulation (DIC) is a clinical entity that is characterized by the systemic activation of coagulation pathways, which can result in organ failure via the generation of fibrin clots and may result in clinical bleeding because of the consumption of platelets and coagulation factors. ${ }^{1-3}$ DIC is reported to have a $20-40 \%$ incidence among patients with severe sepsis. ${ }^{1-3}$ In patients with DIC, the disease progresses rapidly, and the outcome is poor with a 30 $40 \%$ mortality rate because of the presence of severe underlying diseases such as infection, sepsis, malignancy, collagen disease, trauma, and hematological disease. ${ }^{1-3}$ Treatment of the underlying diseases of DIC is essential. Moreover, supportive modalities such as the administration of platelet concentrates, fresh frozen plasma, heparin, activated protein $\mathrm{C}$ 
(APC), protease inhibitors, and antithrombin play an important role in controlling DIC. . $^{1-5}$ However, owing to the absence of randomized controlled trials, a standard treatment has not been established. Regarding the crucial role of APC and the thrombomodulin (TM) system in the pathogenesis of DIC, new agents against DIC, such as recombinant soluble thrombomodulin (rTM), have been developed, and these agents are effective in the treatment of DIC. ${ }^{6-9}$ Here, we report the clinical characteristics and outcomes of 35 DIC cases at our institution.

\section{PATIENTS AND METHODS}

To elucidate the clinical characteristics of DIC, such as laboratory data, diagnosis, side effects, and treatment outcome, we retrospectively analyzed 35 DIC patients (19 men and 16 women) at our institution over a 2-year period from 2008 to 2009. The clinical characteristics of infectious diseases are summarized in Table 1. The patients' age at diagnosis ranged from 2 to 83 years (mean age: 55 years). The causes of DIC were found to be infections (21 cases) such as sepsis (5 cases), chronic active Epstein-Barr virus infection
(CAEBV; 2 cases), pneumonia ( 2 cases), meningitis (2 cases), and acute pancreatitis ( 1 case). The clinical characteristics of hematological diseases are also summarized in Table 1. The patients' age at diagnosis ranged from 21 to 71 years (mean age: 46 years). The causes of DIC were found to be hematological diseases (14 cases) such as acute myelocytic leukemia (AML; 11 cases), acute lymphocytic leukemia (ALL; 2 cases), and chronic lymphocytic leukemia (CLL; 1 case). Diagnosis of DIC was based on the criteria of the Japanese Ministry of Health and Welfare (JMHW), which consist of the underlying diseases, clinical presentation, thrombocytopenia, increased prothrombin time (PT) ratio, decreased fibrinogen, and elevated fibrinogen and fibrin degradation products (FDP). ${ }^{7,10}$ DIC was diagnosed when the DIC score exceeded 7 or 4 points in the presence of severe thrombocytopenia because of bone marrow failure. ${ }^{4,7,10}$ In addition to treating the underlying disease, we administered $\mathrm{rTM}$ at a dose of $380 \mathrm{U} / \mathrm{kg} /$ day for 6 consecutive days. In patients with renal insufficiency on hemodialysis, we administered rTM at a dose of $130 \mathrm{U} / \mathrm{kg} /$ day for 6 consecutive days. When DIC could not be controlled, we extended the administration of rTM beyond the 6-day period. Resolution of DIC

Table 1. Clinical characteristics of disseminated intravascular coagulation patients with infectious diseases and hematological diseases

\begin{tabular}{|c|c|c|c|c|}
\hline Clinical data & \multicolumn{2}{|c|}{ Infectious diseases } & \multicolumn{2}{|l|}{ Hematological diseases } \\
\hline No. of cases & 21 & & 14 & \\
\hline Age (range) at onset & $55 \pm 24(2-8$ & & $46 \pm 19(21-71)$ & \\
\hline Sex, $\mathrm{m} / \mathrm{f}$ & $12 / 9$ & & $7 / 7$ & \\
\hline \multirow[t]{5}{*}{ Underlying diseases } & Sepsis & 5 cases & AML (M0, M2, M3, M4, M5a) & 11 cases $(1,3,2,2,3)$ \\
\hline & CAEBV & 2 cases & ALL (L2) & 2 cases (2) \\
\hline & Pneumonia & 2 cases & CLL & 1 case \\
\hline & Meningitis & 2 cases & & \\
\hline & Others & 10 cases & & \\
\hline Bleeding symptom & 5 & & 6 & \\
\hline Organ symptom & 14 & & 10 & \\
\hline Platelet & $3.7 \pm 2.2(0$ & $9.5)$ & $4.9 \pm 3.8(1.2-14.4)$ & \\
\hline PT ratio & $1.5 \pm 0.7$ & 4.3) & $1.4 \pm 0.3(1.0-2.4)$ & \\
\hline APTT & $60.1 \pm 2.2$ & $6-115.2)$ & $36.9 \pm 6.2(28.2-45.5)$ & \\
\hline Fib & $318.6 \pm 165$ & $(96.5-844.4)$ & $229.6 \pm 147.9(30-568.8)$ & \\
\hline FDP & $77.7 \pm 87.3$ & $9-375.2)$ & $116.7 \pm 212.1(5.1-814.4)$ & \\
\hline CRP & $16.3 \pm 12.8$ & .03-45.4) & $5.5 \pm 7.1(0.05-26.44)$ & \\
\hline $\begin{array}{l}\text { Transfusion of PC during the } \\
\text { treatment with rTM }\end{array}$ & $19.5 \pm 21.3$ & $-60)$ & $30.0 \pm 39.0(0-110)$ & \\
\hline $\begin{array}{l}\text { Transfusion of FFP during the } \\
\text { treatment with rTM }\end{array}$ & $4.4 \pm 6.1(0$ & & $11.8 \pm 13.1(0-35)$ & \\
\hline DIC score (JMHW) & $7.6 \pm 1.6(6$ & & $5.2 \pm 1.8(4-9)$ & \\
\hline $\begin{array}{l}\text { Resolution of DIC at } 7 \text { day (DIC } \\
\text { score) }\end{array}$ & $61.9 \%(13 / 2$ & & $57.1 \%(8 / 14)$ & \\
\hline Outcome & $76.2 \%(16 / 2$ & & $85.7 \%(12 / 14)$ & \\
\hline
\end{tabular}

no., number; f, female; m, male; CAEBV, chronic active Epstein-Barr virus infection; AML, acute myelocytic leukemia; ALL, acute lymphocytic leukemia; CLL, chronic lymphocytic leukemia; PT, prothrombin time; APTT, activated partial thromboplastin time; Fib, fibrinogen; FDP, fibrinogen and fibrin degradation products; CRP, C-reactive protein; PC, platelet concentrates; rTM, recombinant human soluble thrombomodulin; FFP, fresh frozen plasma; DIC, disseminated intravascular coagulation; JMHW, Japanese Ministry of Health and Welfare.

Etiologies of others were consist of pancreatitis, cellulitis, invasive group A streptococcal disease, brain abscess, cholecystitis, acute obstructive suppurative cholangitis, liver abscess, pyelonephritis, peritonitis, systemic lupus erythematosus, and colitis. 
was evaluated by the DIC score on the basis of the criteria of the JMHW at 7 days after the administration of rTM. ${ }^{7}$ Resolution of DIC without severe thrombocytopenia because of bone marrow failure was defined as a score of $\leq 5$ points. $^{7}$ Resolution of DIC with severe thrombocytopenia because of bone marrow failure was defined as a score of $\leq 2$ points. ${ }^{7}$ The treatment outcome was evaluated by the survival rate at 28 days after the infusion of rTM. The exclusion criterion was active life-threatening bleeding with shock. We also evaluated the changes of the coagulation marker and the DIC score by using the Wilcoxon signed-rank test. This retrospective study was conducted in compliance with good clinical practices and the ethical principles of the Declaration of Helsinki. Prior approval was obtained from the ethics review boards at our institution.

\section{RESULTS}

\section{Patient characteristics, laboratory findings, and treatment outcomes in infectious diseases}

The clinical characteristics of infectious diseases are summarized in Table 1. Clinical manifestations such as bleeding (5 cases) and organ dysfunction (14 cases) were observed. The DIC score for infectious diseases ranged from 6 to 11 (mean score: 7.6). We compared the changes in the platelet count (Plt), PT ratio, fibrinogen level (Fib), and FDP to evaluate the efficacy of rTM against DIC (Fig. 1A). The average values of Plt $\left(\times 10^{4} / \mu \mathrm{L}\right)$, the PT ratio, Fib $(\mathrm{mg} / \mathrm{dL})$, and FDP $(\mu \mathrm{g} / \mathrm{mL})$ before the administration of rTM were 3.7, 1.5, 318.6, and 77.7, respectively. The average values of Plt $\left(\times 10^{4} / \mu \mathrm{L}\right)$, the PT ratio, Fib $(\mathrm{mg} / \mathrm{dL})$, and FDP $(\mu \mathrm{g} / \mathrm{mL})$ after treatment were 9.1, 1.2, 329.3, and 24.0, respectively. These findings indicate that Plt, the PT ratio, and FDP are good markers to evaluate the efficacy of DIC treatment. Among these parameters, we evaluated changes in Plt over a 7-day period. The value of Plt gradually increased at 6 days after the administration of rTM (Fig. 1B). These findings demonstrate that the early recovery of Plt was observed through resolution of DIC. Next, we compared the DIC score before and after the administration of rTM (Fig. 2A). The average DIC score before treatment was 7.6, whereas the average DIC score after treatment decreased to 4.9. Resolution of DIC was observed in $61.9 \%(13 / 21)$ of cases. Moreover, 3 of the 8 DIC patients in whom DIC was not resolved at 7 days finally attained resolution at an average of 13.0 days. Consequently, 16 of 21 patients $(76.2 \%)$ were alive with resolution of DIC after the 28-day observation period (Fig. 2B).

\section{Patient characteristics, laboratory findings, and treatment outcomes in hematological diseases}

The clinical characteristics of hematological diseases are summarized in Table 1. Clinical manifestations such as bleeding (6 cases) and organ dysfunction (10 cases) were observed. The DIC score ranged from 4 to 9 for hematological diseases (mean score : 5.2). The average values of Plt ( $\times$ $\left.10^{4} / \mu \mathrm{L}\right)$, the PT ratio, Fib $(\mathrm{mg} / \mathrm{dL})$, and FDP $(\mu \mathrm{g} / \mathrm{mL})$ before treatment with rTM were 4.9, 1.4, 229.6, and 116.7, respectively (Fig. 1C). The average values of Plt $\left(\times 10^{4} / \mu \mathrm{L}\right)$, the PT ratio, Fib $(\mathrm{mg} / \mathrm{dL})$, and FDP $(\mu \mathrm{g} / \mathrm{mL})$ after treatment changed to $7.8,1.2,231.0$, and 30.2 , respectively. These findings indicate that FDP are a good marker to evaluate the efficacy of DIC treatment (Fig. 1C). Moreover, we evaluated the changes in FDP over a period of 7 days. The value of FDP gradually decreased on the third day after administration of rTM (Fig. 1D). These findings indicate that the early recovery of FDP was observed during the resolution of DIC. We compared the DIC score before and after administration of rTM (Fig. 2C). Although the DIC score before the treatment was 5.2, the DIC score after the treatment decreased to 2.7 (Fig. 2C). Resolution of DIC was observed in $57.1 \%(8 / 14)$ of cases. Moreover, 3 of the 6 DIC patients who did not attain resolution of DIC at 7 days finally attained resolution at an average of 11.2 days. Consequently, 12 of the 14 patients were alive (85.7\%) after the 28-day observation period (Fig. 2D).

\section{Treatment outcome of all cases}

Finally, 21 (60.0\%) of 35 DIC patients attained resolution of DIC at 7 days after rTM administration. Furthermore, 7 of the remaining 14 DIC patients (who did not attain resolution at 7 days) attained resolution at an average of 12.1 days. Consequently, $28(80.0 \%)$ of the 35 patients were alive with resolution of DIC after a 28-day observation period. In cases in which the patient did not survive, the cause of death was progression of the underlying disease. Among the 35 DIC cases, $7(70 \%)$ of the 10 DIC patients with severe lifethreatening bleeding symptoms without hemorrhagic shock (oral, gastrointestinal, pulmonary, or abnormal vaginal bleeding) (5 cases in infectious diseases and 5 cases in hematological diseases) for whom treatment with heparin was contraindicated were successfully treated with rTM without the progression of hemorrhage. In 2 patients with renal insufficiency on hemodialysis (1 case, in which there was infectious disease, and 1 case, in which there was hematological disease), we successfully treated them with rTM at a dose of 130 $\mathrm{U} / \mathrm{kg} /$ day. Moreover, when DIC was not controlled, we extended the number of days of rTM administration beyond 6 days [infectious disease group: 9 days ( 1 case), 13 days (1 case), and 16 days ( 1 case); hematological disease group: 13 days $(1$ case)]. No severe side effects, such as thrombosis or bleeding, were observed during the administration of rTM. A skin rash was observed in a single patient. 
A
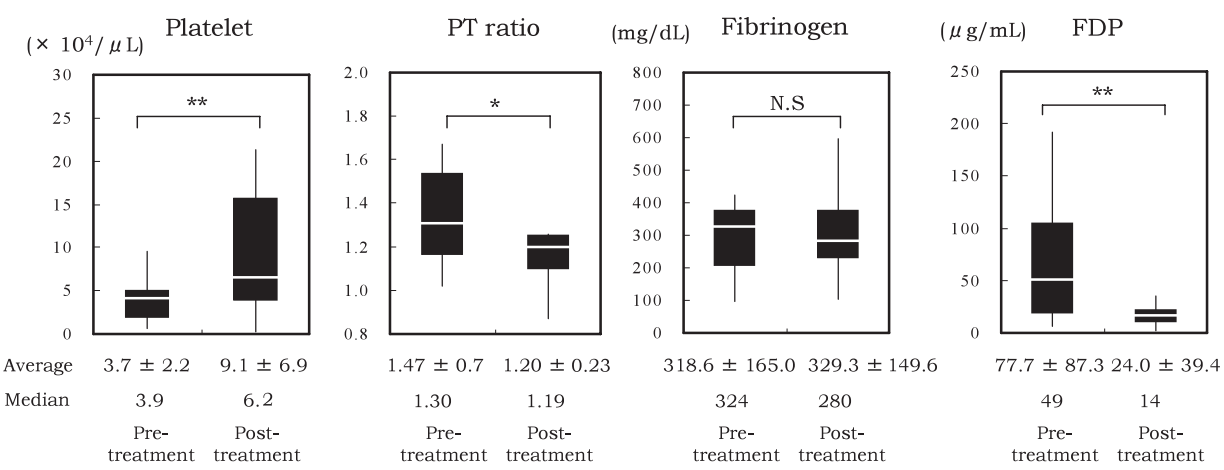

$\mathrm{B}$

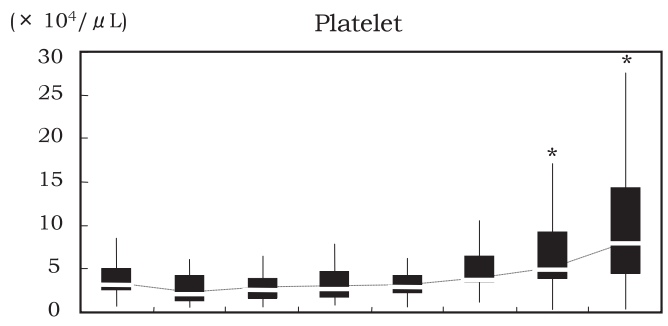

day -1 day 1 day 2 day 3 day 4 day 5 day 6 day 7

Days after treatment with rTM (days)

C
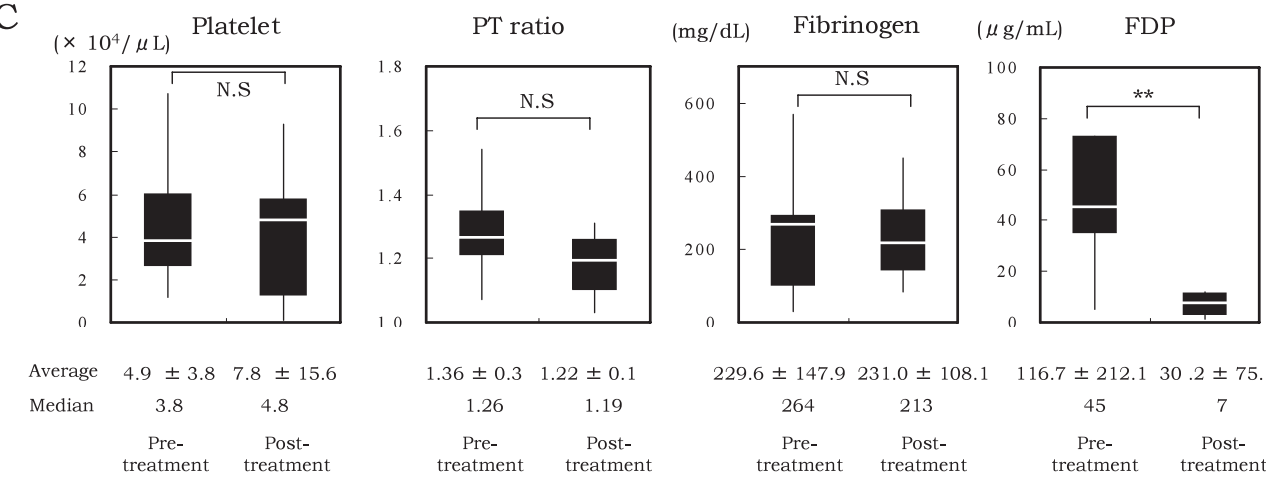

$229.6 \pm 147.9231 .0 \pm 108.1$

$116.7 \pm 212.130 .2 \pm 75.1$

treatment treatment
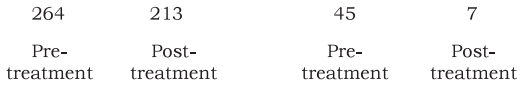

$\mathrm{D}_{(\mu \mathrm{g} / \mathrm{mL})}$

FDP

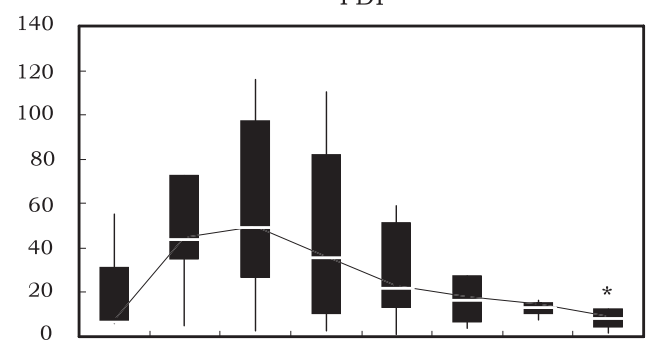

day -1 day 1 day 2 day 3 day 4 day 5 day 6 day 7

Days after treatment with rTM (days)

Fig. 1. The changes in platelet (Plt), the prothrombin time (PT) ratio, fibrinogen level (Fib), and fibrinogen and fibrin degradation products (FDP) before and after the administration of recombinant human soluble thrombomodulin (rTM). (1A) Changes in Plt, the PT ratio, Fib, and FDP before and after the administration of rTM in patients with infectious diseases. (1B) Changes in Plt after the administration of rTM in patients with infectious diseases. (1C) Changes in Plt, the PT ratio, Fib, and FDP before and after the administration of rTM in patients with hematological diseases. (1D) Changes in FDP after the administration of rTM in patients with hematological diseases. **; $\mathrm{P}<0.01, * ; \mathrm{P}<0.05$, compared to pretreatment or day 1 . 
A

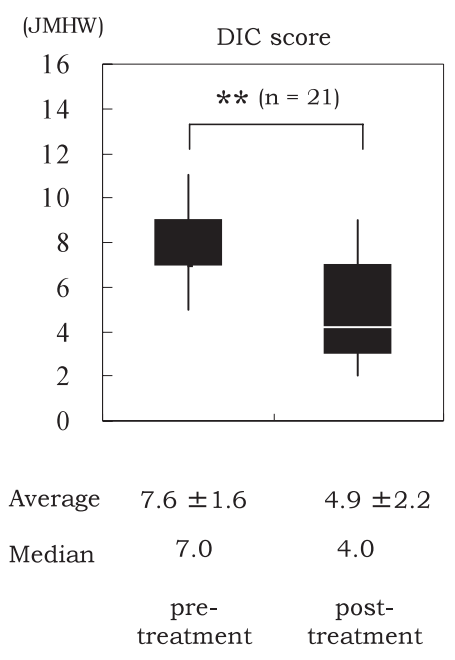

Resolution of DIC at 7 day $(13 / 21=61.9 \%)$

$\mathrm{C}$

(JMHW) DIC score

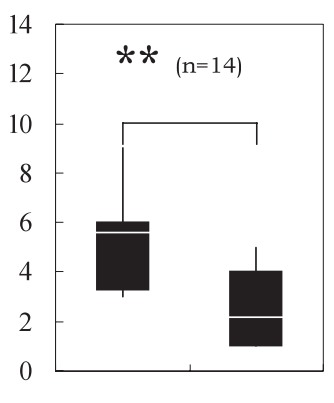

Average

$5.2 \pm 1.8 \quad 2.7 \pm 2.3$

Median

5.5

2.0

pretreatment posttreatment

Resolution of DIC at 7 day $(8 / 14=57.1 \%)$
B (\%)

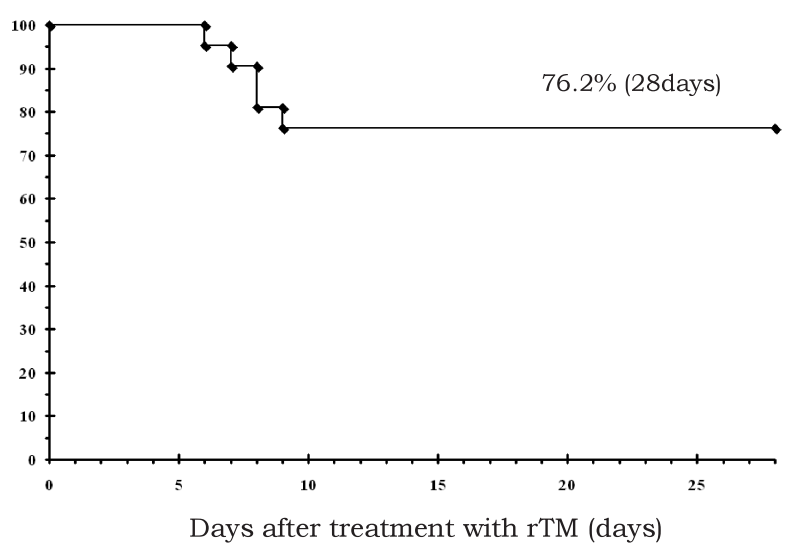

Days after treatment with rTM (days)

$\mathrm{D} \%$

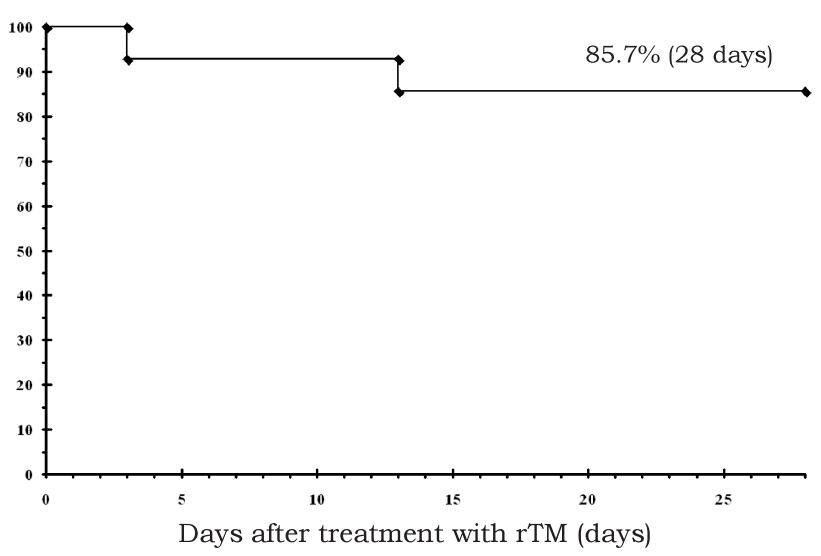

Fig. 2. Changes in the disseminated intravascular coagulation (DIC) score and the outcome of patients with DIC. (2A) Changes in the DIC score before and after the administration of rTM in patients with infectious diseases. (2B) The outcome of patients with DIC with infectious diseases treated by rTM. (2C) Changes in the DIC score before and after the administration of rTM in patients with hematological diseases. (2D) The outcome of patients with DIC and hematological diseases treated by rTM. **; P $<0.01$, compared to pretreatment.

\section{DISCUSSION}

In light of the importance of APC and the TM system in the coagulation process of DIC, rTM was developed as a new effective agent against DIC. ${ }^{6-9}$ TM is a transmembrane protein on the endothelial cell surface and plays important roles in the regulation of intravascular coagulation. ${ }^{6-9}$ Bernard et al. reported that recombinant human APC was effective and safe against severe sepsis in a multicenter randomized controlled trial. ${ }^{5}$ In contrast to the conventional therapeutic agents that have been used against DIC, rTM has anticoagulant and anti-inflammatory effects without any side effects. $^{6-9}$ rTM binds to thrombin to inhibit coagulation. ${ }^{6}$ The thrombin-rTM complex stimulates protein $\mathrm{C}$ to produce APC. ${ }^{6}$ APC inactivates factors VIIIa and Va under protein S. ${ }^{6}$ This pathway halts further thrombin formation. ${ }^{6}$ The anti-inflammatory effect was achieved because rTM suppressed the production of high-mobility group box (HMGB), which is the key cytokine in sepsis. ${ }^{11}$ Iba et al. reported that the combination of antithrombin and rTM improved Plt, Fib, alanine transaminase, lactic dehydrogenase, and HMGB1 values in addition to histological findings in a lipopolysaccharide-induced rat sepsis model. ${ }^{11}$ Saito et al. reported that rTM was effective and safe in a phase III 
randomized double-blind clinical trial of DIC; ${ }^{7}$ they also reported that the resolution rate of DIC was $66.1 \%$ (infection: $66.7 \%$; hematological disease: $65.6 \%$ ) and that the treatment outcome was $78.1 \%$ (infection: $72.0 \%$; hematological disease: $82.8 \%$ ) after a 28 -day observation period. ${ }^{7}$ Therefore, rTM in the phase III study had limitations due to exclusion criteria such as dialysis therapy, serious liver disorders, life-threatening bleeding, and a high probability of developing life-threatening bleeding ; consequently, the indications and contraindications of rTM for DIC in the real world have remained unclear. ${ }^{7}$ In the few available case reports, rTM was successfully administered for DIC with acute myelogenous leukemia in a child, capillary leakage associated with engraftment syndrome, sinusoidal obstructive syndrome, and thrombotic microangiopathy after hematopoietic stem cell transplantation. ${ }^{12-14}$ Therefore, the indication of rTM administration for coagulation abnormalities has gradually expanded, and it is essential to identify the indications and contraindications of coagulation abnormalities.

At our institution, for 7 (70\%) of 10 DIC patients with severe life-threatening bleeding symptoms without hemorrhagic shock (oral, gastrointestinal, pulmonary, or abnormal vaginal bleeding), treatment with heparin was contraindicated; these patients were successfully treated with rTM without progression of hemorrhage. Two patients with renal insufficiency who were undergoing hemodialysis were successfully treated with a reduced dose of rTM. Finally, the treatment outcome was consistent with that of the previous phase III study. These findings clearly suggest that severe life-threatening bleeding without hemorrhagic shock and renal sufficiency in patients undergoing hemodialysis because of DIC is a good indication for rTM.

To the best of our knowledge, our results are the first to reveal that rTM administration may be an effective, safe, and feasible treatment for DIC patients with severe lifethreatening bleeding without hemorrhagic shock and patients with renal insufficiency who are undergoing hemodialysis because of DIC who were excluded in phase III trials, despite the small numbers at our institution.

We speculated that the lack of severe adverse events, such as the progression of bleeding symptoms and severe hemorrhage, was due to the rapid recovery of Plt or FDP in the early days of administration. We suggest that Plt in infectious disease and FDP in hematological disease serve as markers of DIC in various hemostatic abnormal findings. The tendency for the resolution of DIC after the administration of rTM could be observed in the early days in terms of the progression of Plt or FDP. In only 4 cases, we indicated the safety and feasibility of administration with rTM over 6 days to control the disease activity of DIC.

In conclusion, in most of the 35 DIC patients treated at Miyazaki Prefectural Hospital, treatment with rTM was effective. Moreover, no severe complications, such as bleeding, were observed. A randomized study and longer follow-up periods are necessary to determine the group of DIC patients who would benefit the most from treatment with rTM and to elucidate the pathogenesis and treatment of this therapeutic modality.

\section{ACKNOWLEDGEMENTS}

We thank Ms. Sakurai, Ms. Kiyoyama, and Ms. Kugimiya for carefully examining the bone marrow specimens.

\section{REFERENCES}

1 Levi M, Ten Cate H: Disseminated intravascular coagulation. N Engl J Med 341:586-592, 1999

2 Wada H, Asakura H, Okamoto K, Iba T, Uchiyama $\mathrm{T}$, et al.; Japanese Society of Thrombosis Hemostasis/DIC subcommittee. Expert consensus for the treatment of disseminated intravascular coagulation in Japan. Thromb Res 125:6-11, 2009

3 Levi M, Toh CH, Thachil J, Watson HG: Guidelines for the diagnosis and management of disseminated intravascular coagulation. Br J Haematol 145:24-33, 2009

4 Warren BL, Eid A, Singer P, Pillay SS, Carl P, et al.; KyberSept Trial Study Group: Caring for the critically ill patient. High-dose antithrombin III in severe sepsis: A randomized controlled trial. JAMA 286:1869-1878, 2001

5 Bernard GR, Vincent JL, Laterre PF, LaRosa SP, Dhainaut JF, et al.: Efficacy and safety of recombinant human activated protein $\mathrm{C}$ for severe sepsis. N Engl J Med 344:699-709, 2001

6 Maruyama I: Recombinant thrombomodulin and activated protein $\mathrm{C}$ in the treatment of disseminated intravascular coagulation. Thromb Haemost 82:718-721, 1999

7 Saito H, Maruyama I, Shimazaki S, Yamamoto Y, Aikawa N, et al.: Efficacy and safety of recombinant human soluble thrombomodulin (ART-123) in disseminated intravascular coagulation: results of a phase III, randomized, double-blind clinical trial. J Thromb Haemost 5:31-41, 2007

8 Aikawa N, Shimazaki S, Yamamoto Y, Saito H, Maruyama I, et al.: Thrombomodulin alfa in the treatment of infectious patients complicated by disseminated intravascular coagulation : subanalysis from the phase 3 trial. Shock 35:349-354, 2011

9 Ogawa E, Yagasaki H, Kato M, Shichino H, Chin M, et al:: Successful treatment of disseminated intravascular coagulation in a child with acute myelogenous leukaemia using recombinant thrombomodulin. Br J Haematol 149:911-912, 2010

10 Kobayashi N, Maekawa T, Takada M, Tanaka H, Gonmori H: Criteria for diagnosis of DIC based on the analysis of clinical and laboratory findings in 345 DIC patients collected by the Research Committee on DIC in Japan. Bibl Haematol (49):265-275, 1983

11 Iba T, Nakarai E, Takayama T, Nakajima K, Sasaoka T, et al.: Combination effect of antithrombin and recombinant human soluble thrombomodulin in a lipopolysaccharide induced rat sepsis model. Crit Care 13:R203, 2009 
12 Ikezoe T, Takeuchi A, Taniguchi A, Togitani K, Yokoyama A: Recombinant human soluble thrombomodulin counteracts capillary leakage associated with engraftment syndrome. Bone Marrow Transplant 46:616-618, 2011

13 Ikezoe T, Togitani K, Komatsu N, Isaka M, Yokoyama A: Successful treatment of sinusoidal obstructive syndrome after hematopoietic stem cell transplantation with recombinant human soluble thrombomodulin. Bone Marrow Transplant 45:783-785, 2010

14 Inoue Y, Kosugi S, Miura I, Hatta Y, Takeuchi J. Successful treatment of refractory acute GVHD complicated by severe intestinal transplant-associated thrombotic microangiopathy using recombinant thrombomodulin. Thromb Res. 2011 Jan 22. [Epub ahead of print] 